\title{
On the use of semidefinite relaxation for uplink phase-only hybrid beamforming with blockers
}

\author{
Mohammed Reda Bekkar ${ }^{\dagger \ddagger}$, Cyrille Siclet $\ddagger$ Laurent Ros ${ }^{\ddagger}$, Benoit Miscopein ${ }^{\dagger}$, Serge Bories ${ }^{\dagger}$ \\ mohammed-reda.bekkar@cea.fr, benoit.miscopein@cea.fr, serge.bories@cea.fr \\ cyrille.siclet@gipsa-lab.fr laurent.ros@gipsa-lab.fr \\ ${ }^{\dagger}$ CEA-LETI, Minatec campus, Grenoble, France

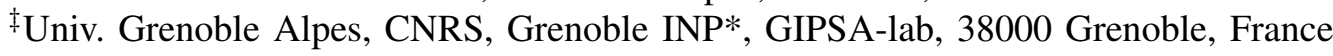 \\ * Institute of Engineering Univ. Grenoble Alpes
}

\begin{abstract}
Densification of mobile networks using small cells (SCs) is a promising approach to achieve the forecasted 1000x growth in capacity for next generation wireless communication systems. However, a major impediment of dense SC network is the low signal-to-interference-plus-noise ratio (SINR) regime, notably in uncoordinated time-division duplexing (TDD) systems in sub-6GHz bands. To circumvent this issue and to enhance the performance of the physical (PHY) layer, hybrid beamforming (HBF) solutions involving both analog and digital stages are necessary. HBF technologies provide interference rejection through analog filtering prior to quantization, spatial multiplexing and require lesser radio-frequency $(\mathrm{RF})$ chains and analog-to-digital converters (ADCs). Conversely, digital beamforming (DBF) performance saturates because of high interferers (blockers) and restricted ADC dynamic range. Moreover, HBF RF circuitry could be further simplified by the use of phase-only weights in analog beamforming (ABF) stage. However, this would be at the cost of making the SINR optimization problem non-convex. Existing sumrate results amalgamating quantization noise consist of lower bounds obtained by distortion factor approximation and worst-case distribution assumption. In this paper, we propose a relaxation approach to phase-only beamforming which allows for a straightforward solution using off-the-shelf interior point algorithms. Rather than directly comparing it in terms of sumrate, we compare it first in terms of SINR, as a function of an algebraic angle between the interferer and the user, with a state-of-the-art local convergence method. Further to this, we include an ADC model to the existing lower bound sumrate results, and analyze various solutions in a sub-6GHz multi-user uplink scenario.
\end{abstract}

Index Terms - hybrid beamforming, optimization, semidefinite programming, sub-6GHz, analog-to-digital converter, small cell.

\section{INTRODUCTION}

The demand for wireless capacity is predicted to increase 1000x times by 2030 compared to the prevailing demands. To overcome this impending challenge, densification of mobile networks using small cells with short coverage as key enablers are deployed. Furthermore, time-division duplexing (TDD) is expedient when considering short coverage, owing to asymmetrical uplink/downlink traffic and the simpler RF front-end realizations. Unfortunately, with densification, the coordination between SCs and frequency reuse strategies become more complex. Thus, to safeguard a given quality-of-service, interSCs interference management is needed. This can be enabled by the use of multi-antenna processing systems on the physical (PHY) layer. In addition, they enable time-frequency resource

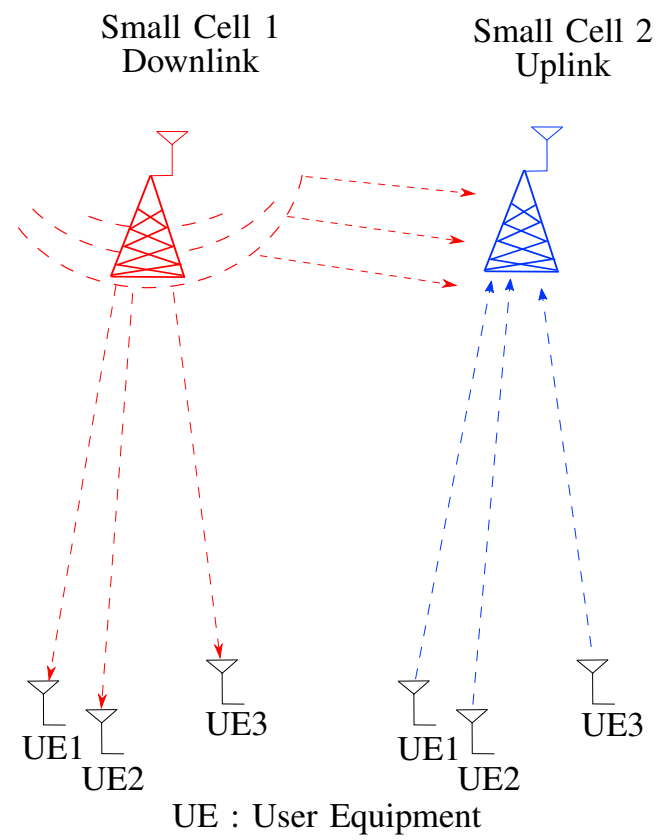

Fig. 1: Typical interference scenario between two adjacent small cells operating in TDD. The SC 1 operating in downlink is jamming SC 2 operating in uplink.

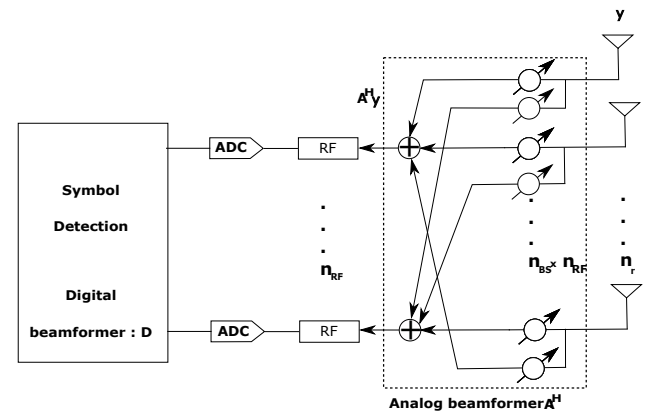

Fig. 2: Full complexity hybrid beamformer architecture. 
sharing between SCs due to the spatial degree of freedom. A typical inter-SC interference scenario is depicted in Fig. 1 where the downlink operating $\mathrm{SC}$ is interfering with the adjacent uplink operating SC.

The millimeter waves (mmW) frequency bands authorize the implementation of multiple-input-multiple-output (MIMO) systems with many antennas, which can concurrently alleviate the high path loss and enable numerous simultaneously supported users through the narrow beam-width beamforming. However, excessive cost, increased energy consumption, and high sensitivity of analog-to-digital converters (ADCs) to blockers [1] make these systems unaffordable and immensely complex [2] for digital implementation. This is further exacerbated by the large number of antennas, increasing the number of RF components (RF chains, mixers etc) linearly.

In contrast, the sub- $6 \mathrm{GHz}$ frequency band does not suffer from a high path loss, leading to a high signal-to-noise ratio (SNR) regime. Nevertheless, in uncoordinated networks inter$\mathrm{SC}$ interference is higher, while the allowed number of antennas is lower. Furthermore, the SCs operating in sub- $6 \mathrm{GHz}$ may be prone to signal blockage during the analog-to-digital conversion due to high interferers (e.g. the scenario of Fig. 1). Without acknowledging the quantization noise introduced by the ADC in the channel model, the benefit of analog spatial filtering present in HBF is not evident.

In $\mathrm{HBF}$, analog circuitry with different complexities are available, directly influence the computing complexity of the analog beamweights. These beamweights can be implemented either using phase shifters only, or using amplifiers/attenuators and phase shifters simultaneously. Various configurations of the beamweight network connection can be realized, varying from the fully connected architecture, to the simpler partially connected architecture [3].

In this work, we shall only consider a fully connected phase shifter only network implementation. As stated previously, HBF allows the use of fewer RF chains and ADCs (compared to the full digital architecture) and spatial filtering of blockers [1] through the ABF stage. Moreover, the lower dimensional digital stage improves the interference rejection and separates the users. In the literature, the beamforming problem for SCs has been addressed via different cost functions, such as the sumrate, the output SINR and the transmitted power, in amalgam with different constraints like minimum SINR, maximum average transmitted power, maximum per-antenna emitted power and phase-only weights in the ABF stage. In [4], Sohrabi and Yu, focus on the problem of maximum sumrate beamforming at the transmitter and receiver with phaseonly constraints in a point-to-point scenario. They indicate that at either the transmitter or the receiver, the problem reduces to a maximum sumrate beamforming problem with per-antenna power constraints addressed in [5]. Wiesel et al. in [6] address the problem of designing a beamformer at the transmitter (precoding), in a multiuser scenario (downlink) with a zero-forcing criterion (maximum signal-to-interferenceratio) and per-antenna power constraints. The authors claim that their solution based on generalized inverses may surpass the pseudo-inverse solution in the dual uplink scenario under noise uncertainty conditions. The novelty of our work lies in the fact that we address the maximum SINR HBF problem at the receiver, with phase-only constraints in a multiuser uplink scenario and unlike [4]-[6], we incorporate ADC model to illustrate the practicality of the hybrid architecture compared to the full digital one. In the sequel, we compare our proposed solution with the state-of-the-art solution of Zhang et al [1] and demonstrate the relevance of the method. In our preceding work [7], we analyzed the SINR loss in existence of a blocker, as a function of the angle of arrival of the blocker for suboptimal approaches, designed on simple phases extraction algorithms. In this paper, we propose to use a semidefinite relaxation (SDR) approach to convert the non-convex problem into a semidefinite programming (SDP) problem, making it easily solvable by off-the-shelf optimization toolboxes. Our contributions are then multi-fold. First, we shall address the problem of a single user phase-only beamforming, and evaluate the SINR loss of different solutions (SDR, Capon and Zhang et al) as a function of a channel orthogonality metric between the user and the interferer. This results in a simple and reliable benchmark of the algorithms that are designed to operate with a low number of antennas and a high probability of non-orthogonal users and interferers channels. Secondly, we consider the the uplink channel system model, and assess the performances of the solutions through a lower bound of the uplink sumrate obtained under some assumptions that allow tractable semi-analytical simulations. Results are obtained assuming perfect channel state information at the receiver.

The paper is organized as follows: single user and multiuser analysis are respectively addressed in section II and in section III. Numerical simulations are presented in section IV and we render the conclusions in section V. We use capital boldface characters and lower case boldface characters to denote matrices and column vectors, respectively. The $N \times N$ identity matrix is noted $\mathbf{I}_{N} \cdot(\cdot)^{H},(\cdot)^{-1}$ denote the conjugate transpose and the matrix inverse respectively. The $(k, m)$-th entry of a matrix $\mathbf{A}$ is noted $[\mathbf{A}]_{k, m}$ and its $(k)$-th column $\mathbf{a}_{(k)}$.

\section{SINGLE-USER, SINGLE-INTERFERER ANALYSIS}

Consider a $n_{r}$ antennas $\mathrm{SC}$, receiving a signal $\mathbf{y} \in \mathbb{C}^{n_{r}}$ composed of a superposition of a single antenna user signal $x_{u} \in \mathbb{C}$ and a single antenna interferer $x_{i} \in \mathbb{C}$. The SC processes the received signal by computing a beamforming vector $\mathbf{w}=\left[w_{1} \ldots w_{n_{r}}\right]^{T}$. The channel model can be written as follows:

$$
\begin{aligned}
\mathbf{y} & =\mathbf{h}_{u} x_{u}+\mathbf{h}_{i} x_{i}+\mathbf{n} \\
\mathbf{w}^{H} \mathbf{y} & =\mathbf{w}^{H} \mathbf{h}_{u} x_{u}+\mathbf{w}^{H} \mathbf{h}_{i} x_{i}+\mathbf{w}^{H} \mathbf{n}
\end{aligned}
$$

where $\mathbf{h}_{u}$ and $\mathbf{h}_{i}$ are respectively the channel vectors of the user and the interferer in $\mathbb{C}^{n_{r}}$ and $\mathbf{n}$ is the thermal noise vector whose elements follow $\mathcal{C N}\left(0, \sigma_{n}^{2}\right)$ and whose covariance matrix is $\mathbf{R}_{\mathbf{n}}=\sigma_{n}^{2} \mathbf{I}$. Without loss of generality we consider that the user emits a power $\mathbb{E}\left[\left|x_{u}\right|^{2}\right]=1$ and 
the interferer $\mathbb{E}\left[\left|x_{i}\right|^{2}\right]=P_{i}$. The instantaneous covariance matrices of the user signal and the interferer signal are defined as $\mathbf{R}_{u}=\mathbf{h}_{u} \mathbf{h}_{u}^{H}$ and $\mathbf{R}_{i}=P_{i} \mathbf{h}_{i} \mathbf{h}_{i}^{H}$ respectively. In these conditions the SINR conditioned to the channel realization can be written as:

$$
\mathrm{SINR}=\frac{\mathbf{w}^{H} \mathbf{R}_{u} \mathbf{w}}{\mathbf{w}^{H}\left(\mathbf{R}_{i}+\mathbf{R}_{n}\right) \mathbf{w}}
$$

In the case of unconstrained choice of the beamforming vector elements, i.e. $w_{k} \in \mathbb{C}$, the maximum SINR solution is known to be the generalized Capon beamformer [8]. It corresponds to the eigenvector $\mathbf{w}_{C}$ associated to the biggest eigenvalue of $\left(\mathbf{R}_{i}+\mathbf{R}_{n}\right)^{-1} \mathbf{R}_{u}$, and its attained SINR is equal to this eigenvalue. The supremum of this eigenvalue can be calculated as the SINR attained by the matched filter while setting $\mathbf{R}_{i}=$ 0 (perfect interference cancellation) i.e. :

$$
\begin{aligned}
\operatorname{SINR}_{\mathrm{MF}} & =\frac{\mathbf{h}_{u}^{H} \mathbf{R}_{u} \mathbf{h}_{u}}{\mathbf{h}_{u}^{H} \mathbf{R}_{n} \mathbf{h}_{u}}=\frac{\left\|\mathbf{h}_{u}\right\|^{2}}{\sigma_{n}^{2}} \\
\lambda_{\max } & =\max \left\{\operatorname{eig}\left(\mathbf{R}_{i}+\mathbf{R}_{n}\right)^{-1} \mathbf{R}_{u}\right\}=\frac{\mathbf{w}_{C}^{H} \mathbf{R}_{u} \mathbf{w}_{C}}{\mathbf{w}_{C}^{H}\left(\mathbf{R}_{i}+\mathbf{R}_{n}\right) \mathbf{w}_{C}} \\
\lambda_{\max } & \leq \operatorname{SINR}_{\mathrm{MF}}
\end{aligned}
$$

It is clear that $\lambda_{\max }$ depends on the channel vectors realization. The legitimate questions here are: when does the equality to its supremum hold, and how much loss is introduced when the equality does not hold? To answer these questions we characterize the channel realization by the angle $\alpha$ between the user and the interferer channel vectors:

$$
\cos (\alpha)=\frac{\boldsymbol{R e}\left(\mathbf{h}_{u}^{H} \mathbf{h}_{i}\right)}{\left\|\mathbf{h}_{u}\right\|\left\|\mathbf{h}_{i}\right\|}
$$

The rationale behind this is that the interferer channel vector can always be decomposed into the sum of a usersubspace-component and an orthogonal-to-the-user-subspacecomponent:

$$
\mathbf{h}_{i}=\mathbf{h}_{i}^{u}+\mathbf{h}_{i}^{u \perp}
$$

The interference is harder to cancel as the $\left\|\mathbf{h}_{i}^{u}\right\|$ grows relatively to $\left\|\mathbf{h}_{i}^{u \perp}\right\|$ or equivalently as $\cos (\alpha)$ approaches 1 .

\section{A. SDR approach to the optimal SINR phase-only beamform- ing problem}

In the phase-only case the optimal SINR beamforming problem can be formulated as [1]:

$$
\begin{aligned}
\operatorname{maximize} & \frac{\mathbf{w}^{H} \mathbf{R}_{u} \mathbf{w}}{\mathbf{w}^{H}\left(\mathbf{R}_{i}+\mathbf{R}_{n}\right) \mathbf{w}} \\
\text { s.t. } & \mathbf{w} \in \mathcal{C} \mathcal{M}
\end{aligned}
$$

where $\mathcal{C} \mathcal{M}=\left\{\mathbf{w} \in \mathbb{C}^{n_{r}}|\quad| w_{k}|=| w_{1} \mid \quad \forall k=2 \ldots n_{r}\right\}$ is the set of constant modulus vectors. Since this set is nonconvex the optimization problem stated above is also nonconvex and no analytical solution is available. Consequently we propose the use of an SDR strategy to find a solution to this problem [9]. First we reformulate the problem similarly to the Capon approach and add the constant modulus constraint using the covariance matrix of the received signal conditioned to the channel realization $\mathbf{R}_{\mathbf{y}}=\left(\mathbf{R}_{u}+\mathbf{R}_{i}+\mathbf{R}_{n}\right)$ :

$$
\begin{aligned}
\operatorname{minimize} & \mathbf{w}^{H} \mathbf{R}_{\mathbf{y}} \mathbf{w} \\
\text { s.t. } & \mathbf{w}^{H} \mathbf{h}_{u}=1 \\
& \mathbf{w} \in \mathcal{C} \mathcal{M}
\end{aligned}
$$

Secondly, we transform the constraint $\mathbf{w}^{H} \mathbf{h}_{u}=1$ into a power constraint $\mathbf{w}^{H} \mathbf{R}_{u} \mathbf{w}=1$ and use the following identities:

$$
\begin{aligned}
\mathbf{w}^{H} \mathbf{R}_{\mathbf{y}} \mathbf{w} & =\operatorname{Tr}\left(\mathbf{w}^{H} \mathbf{R}_{\mathbf{y}} \mathbf{w}\right)=\operatorname{Tr}\left(\mathbf{R}_{\mathbf{y}} \mathbf{w} \mathbf{w}^{H}\right) \\
\mathbf{w}^{H} \mathbf{R}_{u} \mathbf{w} & =\operatorname{Tr}\left(\mathbf{w}^{H} \mathbf{R}_{u} \mathbf{w}\right)=\operatorname{Tr}\left(\mathbf{R}_{u} \mathbf{w} \mathbf{w}^{H}\right)
\end{aligned}
$$

Finally, by defining $\mathbf{W}=\mathbf{w} \mathbf{w}^{H}$, a hermitian positive semidefinite matrix, we formulate the problem as follows:

$$
\begin{array}{cl}
\operatorname{minimize} & \operatorname{Tr}\left(\mathbf{R}_{\mathbf{y}} \mathbf{W}\right) \\
\text { s.t. } & \operatorname{Tr}\left(\mathbf{R}_{u} \mathbf{W}\right)=1 \\
& W_{k k}=W_{11} \quad \forall k=2 \ldots n_{r} \\
& \mathbf{W} \succeq 0 \\
& \operatorname{rank}(\mathbf{W})=1
\end{array}
$$

It is interesting to note that in this latter problem the nonconvex constraint is only the rank constraint, and dropping it relaxes the problem into the following SDP convex problem:

$$
\begin{aligned}
\operatorname{minimize} & \operatorname{Tr}\left(\mathbf{R}_{\mathbf{y}} \mathbf{W}\right) \\
\text { s.t. } & \operatorname{Tr}\left(\mathbf{R}_{u} \mathbf{W}\right)=1 \\
& W_{k k}=W_{11} \quad \forall k=2 \ldots n_{r} \\
& \mathbf{W} \succeq 0
\end{aligned}
$$

This latter problem is convex and easily solvable by an interior point solver, using the CVX MATLAB [10] toolbox for example. Once a solution $\mathbf{W}^{*}$ to the problem (21) is found, one must convert it into a feasible solution $\mathrm{w}^{*}$ of the problem (11). Several methods exist, they would generally introduce a loss of optimality (otherwise we would have solved an NPHard problem by a polynomial time algorithm) [11]. However for certain specially structured problems there is no loss of optimality. This is for example the case if $\operatorname{rank}\left(\mathbf{W}^{*}\right)=1$, which means that there exists only one dimension along which it is possible to extract a candidate solution $\mathbf{w}^{*}$. In our case we use the eigenvector associated to the maximum eigenvalue of $\mathbf{W}^{*}$, i.e. if the ordered eigenvalues are $\lambda_{1} \geq \ldots \lambda_{n_{r}}$ :

$$
\begin{aligned}
\mathbf{W}^{*} & =\sum_{i=1}^{n_{r}} \lambda_{i} \mathbf{e}_{i} \mathbf{e}_{i}^{H} \\
\mathbf{w}^{*} & =\sqrt{\lambda_{1}} \mathbf{e}_{1}
\end{aligned}
$$

Please note that if $\operatorname{rank}\left(\mathbf{W}^{*}\right)>1$ this heuristic does not generally give the optimal solution. But we observed from our simulations that the rank of $\mathbf{W}$ is always one and it seems that the solution found by this heuristic is optimal. Nevertheless the attained SINR is not necessarily the optimum attained by the Capon beamformer without beamweights constraints. 


\section{MUlTiUSER SYSTEM MODEL}

In this section we consider the multiuser model and its associated HBF algorithm in the case of phase-only weights in the analog stage [1]. We also introduce ADCs into the model.

Consider an $n_{r}$ antennas SC receiving a signal $\mathbf{y} \in \mathbb{C}^{n_{r}}$ composed of a superposition of $n_{u}$ single antenna users signal $\mathbf{x} \in \mathbb{C}^{n_{u}}$ and $n_{b}$ single antenna blockers $\mathbf{x}_{b} \in \mathbb{C}^{n_{b}}$. The users channels matrix $\mathbf{H}$ and the blockers channel matrix $\mathbf{H}_{b}$ are respectively in $\mathbb{C}^{n_{r} \times n_{u}}$ and $\mathbb{C}^{n_{r} \times n_{b}}$. Without loss of generality we assume that the users emit a unit power, i.e. $\mathbb{E}\left[\left|x_{k}\right|^{2}\right]=$ 1 and the blockers emit a power equal to $\mathbb{E}\left[\left|x_{b k}\right|^{2}\right]=P_{b k}$. The covariance matrices of the users signal and the blockers signal conditioned to the channels realization are respectively $\mathbf{R}_{m u}=\mathbf{H H}^{H}$ and $\mathbf{R}_{b}=\mathbf{H}_{b} \operatorname{diag}\left\{P_{b 1} \ldots P_{b n_{b}}\right\} \mathbf{H}_{b}^{H}$. The SC station first processes this signal by an analog stage, modeled by an $\mathrm{ABF}$ matrix $\mathbf{A} \in \mathcal{C} \mathcal{M}^{n_{R F}}$, with output $\mathbf{y}_{a}=\mathbf{A}^{H} \mathbf{y}$. Secondly the quantization step is modeled by a non linear function $\mathcal{Q}($.$) that acts as a scalar quantizer on the real and$ imaginary parts of every element of $\mathbf{y}_{a}$, yielding to the vector $\mathbf{y}_{q}$. Finally, the DBF step is performed using the matrix $\mathbf{D} \in$ $\mathbb{C}^{n_{R F} \times n_{u}}$. These steps are summarized as follow:

$$
\begin{aligned}
\mathbf{y} & =\mathbf{H x}+\mathbf{H}_{B} \mathbf{x}_{B}+\mathbf{n} \\
\mathbf{y}_{a} & =\mathbf{A}^{H} \mathbf{y} \\
\mathbf{y}_{q} & =\mathcal{Q}\left(\mathbf{y}_{a}\right) \\
\hat{\mathbf{x}} & =\mathbf{D}^{H} \mathbf{y}_{q}
\end{aligned}
$$

\section{A. ADC model assumptions}

Due to the quantization step being a nonlinear operation, we make some assumptions to make the sumrate expression and DBF matrix construction tractable. First the ADC output can be rewritten by using an additive quantization noise model (AQNM) [12]

$$
\mathbf{y}_{q}=(1-\rho) \mathbf{y}_{a}+\mathbf{n}_{q}
$$

which introduces the distortion factor $\rho$. By assuming gaussian distributions for users and interferers signals, and a Max-Lloyd quantizer (the optimal non-uniform quantizer) the distortion factor can be approximated using the Pater-Dite formula [13]:

$$
\rho \approx \frac{\pi \sqrt{3}}{2} 2^{-2 b}
$$

for a number of $b$ bits greater or equal to 3 [14]. Finally the covariance matrices of the quantization noise and the ADCs outputs are [12]

$$
\begin{aligned}
& \mathbf{R}_{\mathbf{y}_{q}} \approx(1-\rho)\left((1-\rho) \mathbf{R}_{\mathbf{y}_{a}}+\rho \operatorname{diag}\left(\mathbf{R}_{\mathbf{y}_{a}}\right)\right. \\
& \mathbf{R}_{\mathbf{n}_{q}}=\mathbf{R}_{\mathbf{y}_{q}}-(1-\rho)^{2} \mathbf{R}_{\mathbf{y}_{a}}
\end{aligned}
$$

\section{B. Analog beamforming matrix construction}

In this paper we restrict to the case of $n_{u}=n_{R F}$ by assigning one RF chain to each user. We also use a user disjoint approach, which means that every column $\mathbf{a}_{k}$ of $\mathbf{A}$ is calculated independently from the others by solving the problem (21) while setting:

$$
\begin{aligned}
\mathbf{W} & =\mathbf{a}_{k} \mathbf{a}_{k}^{H} \\
\mathbf{R}_{u} & =\mathbf{h}_{k} \mathbf{h}_{k}^{H} \\
\mathbf{R}_{\mathbf{y}} & =\mathbf{R}_{m u}+\mathbf{R}_{b}+\mathbf{R}_{\mathbf{n}}
\end{aligned}
$$

\section{Digital beamforming matrix construction}

Once an ABF matrix is found, it is possible to calculate a Capon DBF matrix as [8]:

$$
\mathbf{D}=\mathbf{R}_{\mathbf{y}_{q}}^{-1} \mathbf{A}^{H} \mathbf{H}
$$

which consists of the product of the inverse of the quantized signal's autocorrelation matrix and the equivalent channel matrix $\mathbf{A}^{H} \mathbf{H}$.

\section{Uplink sumrate lower bound in the presence of $A D C$}

The entropy of a multivariate gaussian distribution with an autocorrelation matrix $\mathbf{R}$ is $\log _{2} \operatorname{det}(2 \pi e \mathbf{R})$. This formula is used to calculate the well known information theory result [15] of the maximum uplink sumrate (multiple access channel):

$$
\sum_{k=1}^{n_{u}} R_{k}=I(\mathbf{y}, \mathbf{x})=\log _{2} \operatorname{det}\left(\mathbf{I}+\left(\mathbf{R}_{b}+\mathbf{R}_{\mathbf{n}}\right)^{-1} \mathbf{H} \mathbf{H}^{H}\right)
$$

This result assumes a gaussian thermal noise, a gaussian users signal and a gaussian blockers signal, without considering any beamforming nor quantization. However if we rewrite our channel model according to the assumptions of III-A:

$$
\begin{aligned}
\hat{\mathbf{x}} & =\mathbf{D}^{H}\left((1-\rho)\left(\mathbf{A}^{H}\left(\mathbf{H x}+\mathbf{H}_{b} \mathbf{x}_{b}+\mathbf{n}\right)\right)+\mathbf{n}_{q}\right) \\
& =\mathbf{H}_{t o t} \mathbf{x}+\mathbf{n}_{t o t} \\
\mathbf{R}_{\mathbf{n}_{t o t}} & =(1-\rho)^{2}\left(\mathbf{A}^{H} \mathbf{R}_{\mathbf{y}} \mathbf{A}\right)+\mathbf{D}^{H} \mathbf{R}_{\mathbf{n}_{q}} \mathbf{D}
\end{aligned}
$$

the mutual information $I(\hat{\mathbf{x}}, \mathbf{x})$ is analytically non-tractable because of the unknown quantization noise distribution and its entropy. Nevertheless a lower bound on the sumrate can be derived taking the worst possible quantization noise distribution for a given variance, namely the gaussian distribution [12] [16] [17]:

$$
R_{\text {sum }}^{L B}=\log _{2} \operatorname{det}\left(\mathbf{I}+\mathbf{R}_{\mathbf{n}_{t o t}}^{-1} \mathbf{H}_{t o t} \mathbf{H}_{\text {tot }}^{H}\right)
$$

\section{Simulations Results}

In this section, we present our numerical results to compare between HBF solutions in the frames presented in the previous sections. Namely comparing the SINRs in a single-user singleinterferer scenario, and comparing the sumrate in a multiuser uplink scenario in presence of blockers. For single user simulations we use a stochastic channel model. This allows the construction of the interferer channel according to eq.(8) and fixing $\cos (\alpha)$ for every run. For multi-user simulations we use a more realistic geometry based channel model with uniform linear array assumption (ULA) for the SC receiver. We assumed unit emitted power for users and variable emitted power for blocker/interferer, expressed relatively to the users power. 


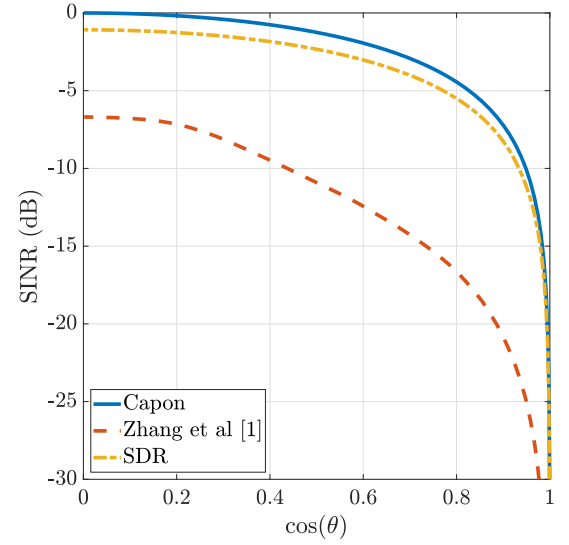

Fig. 3: SINR of different beamforming solutions relative to the matched filter SINR with perfect interference rejection.

\section{A. Single user SINR simulations}

We compare the mean SINR of our phase-only beamforming algorithm and a state of the art algorithm [1] relatively to the matched filter supremum reference described in II. Both algorithms are initialized with the Capon solution phases.

Their respective performance is plotted as a function of $\cos (\alpha)$ in Fig.3. Simulations were performed using $n_{r}=8$, $P_{i}=40 \mathrm{~dB}$ and a high $\mathrm{SNR}=20 \mathrm{~dB}$. For a fair comparison termination criteria are set to $10^{-6}$ in terms of linear SINR progression and to 100 in terms of maximum number of iterations in both algorithms. The elements of $\mathbf{h}_{u}$ were generated following $\mathcal{C N}(0,1)$ and then normalized to have $\left\|\mathbf{h}_{u}\right\|^{2}=n_{r}$ to keep an SNR gain compared to a single antenna system. $\mathbf{h}_{i}$ is calculated from each realization and each $\cos (\theta)$ point. We notice a considerable improvement up to $10 \mathrm{~dB}$ of the SINR as compared to the local convergence approach of [1]. We also notice that the relaxation approach does not attain the optimum unconstrained Capon (amplitude and phase weights tuning) level, which is due to the degree of freedom reduction. We conclude that the SDR performs better than the power iteration method of [1] due to the convex reformulation and the rank one solution allowed by the special structure of the problem.

\section{B. Multiuser sumrate simulations}

In this section, we compare the mean sumrate of our phaseonly beamforming algorithm to the state-of-the-art algorithm [1] initialized with the Capon solution phases. In addition we plot also the Capon beamformer sumrate in the case of a hybrid system (amplitude and phase, in the analog and in the digital stage) and a full digital system. Simulations were performed using $n_{r}=16, n_{u}=8, n_{b}=1$ and variable $P_{b} \in[0,60] \mathrm{dB}$ and $b \in\{4,8,12\}$ bits. Termination criteria and number of iterations are set equally to the previous subsection. Channel matrices are generated following an ULA single path model, i.e. their columns $\mathbf{h}_{k}=h\left[1, e^{-j 2 \pi d \sin (\theta)} \ldots e^{-j\left(n_{r}-1\right) 2 \pi d \sin (\theta)}\right]^{T}$ with the interelement distance $d=0.5$, the fading $h \sim \mathcal{C N}(0,1)$ and the

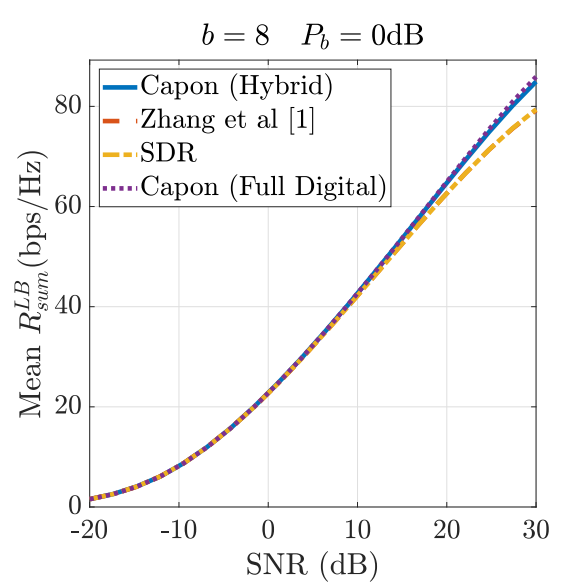

(a)

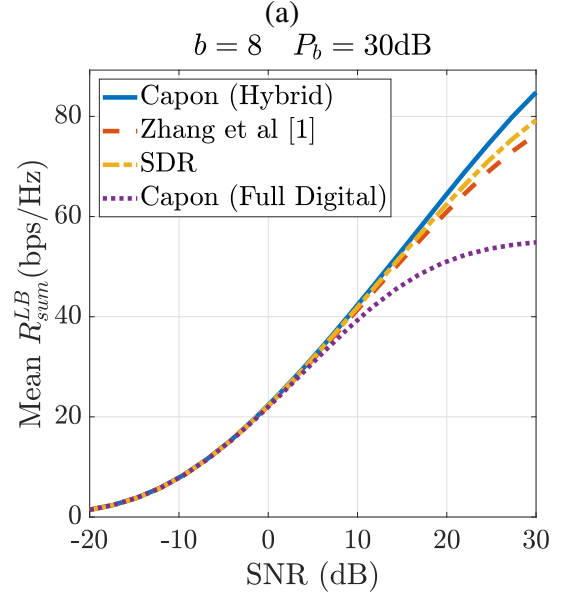

(b)

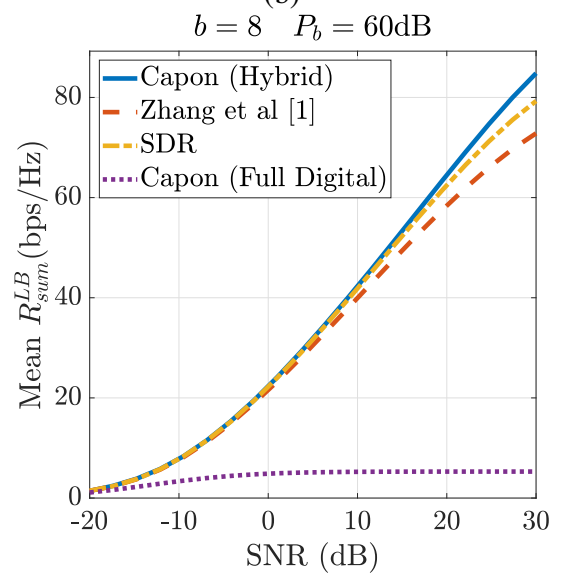

(c)

Fig. 4: Sumrate lower bound of different HBF solutions for $b=8$ bits and variable blocker power $P_{b}$.

path angle of arrival $\theta$ following a uniform law in $\left[-90^{\circ}, 90^{\circ}\right]$.

In Fig. 4a, we observe that 8 bits is sufficient for all the schemes to properly operate when the blocker is as high as the users power (i.e. $0 \mathrm{~dB}$ ). Nonetheless a clear performance degradation of the full digital scheme appears as the blocker power increases in Fig. 4b, due to the non-negligible quantiza- 


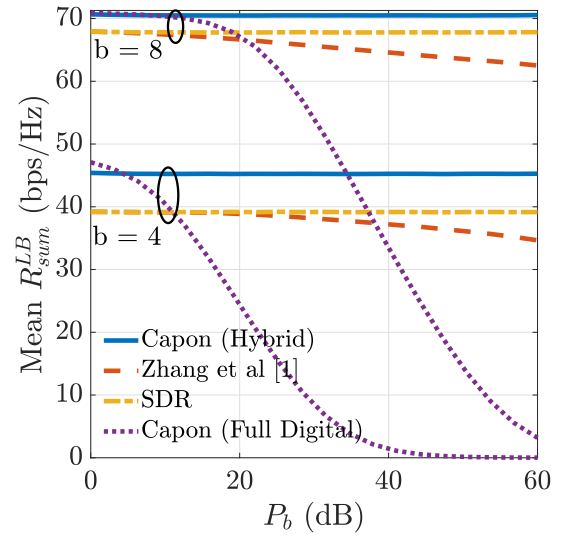

Fig. 5: Sumrate lower bound of different HBF at $\mathrm{SNR}=20 \mathrm{~dB}$ as a function of the blocker power.

tion noise introduced by the high blocker. The use of an analog spatial filtering stage is thus necessary to avoid an extremely low sumrate. Conversely, HBF schemes keep a near optimal performance with a slight advantage of SDR in the high SNR regime.

In Fig. 5 we plot the mean sumrate at $\mathrm{SNR}=20 \mathrm{~dB}$ as a function of the blocker power for $b=4$ and $b=8$ bits. We notice again a clear performance diminution of the full digital scheme as the blocker power increases, worsened by passing from $b=8$ to $b=4$ bits. The hybrid Capon scheme and the SDR scheme do not depend on the blocker power due to a perfect interference nulling. The loss between the two schemes arises from the white noise gain introduced by the phase-only restriction (since introducing a null in the radiation pattern increases the secondary lobes levels, and thus the white noise gain [18]). The hybrid architecture is unable to apodize the secondary lobes due to the constant modulus constraint. This higher white noise gain increases the quantization noise power during the digitization step. Comparatively the local convergence nature of the method [1] implies an imperfect null alignment and thus a sensitivity to the blocker power.

\section{CONClusion}

In this paper, we addressed the problem of phase-only beamforming for the analog stage in HBF architecture. We used an existing relaxation approach to convert the non-convex problem into an SDP problem, making it easily solvable by off-the-shelf optimization toolboxes. In contrast to a state-ofthe-art solution, this method has a guaranteed convergence to the optimum SINR. The existing results on the sumrate taking into account the ADC quantization noise consist of lower bounds obtained under worst-case distribution hypothesis and approximated distortion factor. Rather than directly comparing in terms of sumrate, we preferred to compare the algorithms in terms of SINR first, as a function of a user-to-interferer algebraic angle. This created a more reliable benchmark in a high SNR single user scenario. We then adapted literature results to evaluate the sumrate in a multiuser TDD scenario of a sub-6GHz SC operating in uplink, with blocker level up to
$60 \mathrm{~dB}$. We noticed that the full digital scheme experiences a rapid saturation and even a zero sumrate in the worst blocking cases with fewer ADCs bits, whereas hybrid schemes keep an acceptable performance with low loss to optimality. In future works, performance analysis will be extended to multi-path channel model and a limited resolution phase shifters.

\section{ACKNOWLEDGMENT}

The authors wish to acknowledge Ali Waqar AZIM for the helpful comments and remarks.

\section{REFERENCES}

[1] X. Zhang, M. Coldrey, T. Eriksson, and M. Viberg, "Hybrid beamforming in uplink massive MIMO systems in the presence of blockers," in 2017 IEEE Int. Conf. on Acoustics, Speech and Signal Process. (ICASSP), Mar. 2017, pp. 6503-6507.

[2] Y.-C. Ko and M.-J. Kim, "Channel estimation and analog beam selection for uplink multiuser hybrid beamforming system," EURASIP Journal on Wireless Commun. and Networking, vol. 2016, no. 1, Dec. 2016.

[3] A. F. Molisch, V. V. Ratnam, S. Han, Z. Li, S. L. H. Nguyen, L. Li, and K. Haneda, "Hybrid Beamforming for Massive MIMO: A Survey," IEEE Commun. Mag., vol. 55, no. 9, pp. 134-141, 2017.

[4] F. Sohrabi and W. Yu, "Hybrid digital and analog beamforming design for large-scale MIMO systems," in IEEE Int. Conf. on Acoustics, Speech and Signal Process., Apr. 2015, pp. 2929-2933.

[5] Z. Pi, "Optimal transmitter beamforming with per-antenna power constraints," in IEEE Int. Conf. on Commun., Jun. 2012, pp. 3779-3784.

[6] A. Wiesel, Y. C. Eldar, and S. Shamai, "Zero-Forcing Precoding and Generalized Inverses,' IEEE Trans. on Signal Process., vol. 56, no. 9, pp. 4409-4418, Sep. 2008.

[7] M. R. Bekkar, B. Miscopein, S. Bories, L. Ros, and C. Siclet, "Constant modulus hybrid beamforming for multi-user systems in the presence of blockers," in Int. Conf. on Telecommun., Jun. 2018, pp. 378-382.

[8] A. Hassanien, S. Shahbazpanahi, and A. B. Gershman, "A generalized capon estimator for localization of multiple spread sources," IEEE Trans. on Signal Process., vol. 52, no. 1, pp. 280-283, Jan. 2004.

[9] C. Lu, W. Sheng, Y. Han, and X. Ma, "Robust adaptive phase-only beamforming algorithm for interference suppression," Int. Journal of Adaptive Control and Signal Process., vol. 29, no. 9, pp. 1152-1164, Sep. 2015. [Online]. Available: http://doi.wiley.com/10.1002/acs.2527

[10] M. Grant and S. Boyd, "CVX: Matlab software for disciplined convex programming, version 2.1," http://cvxr.com/cvx, Mar. 2014.

[11] Z. Q. Luo, W. K. Ma, A. M. C. So, Y. Ye, and S. Zhang, "Semidefinite Relaxation of Quadratic Optimization Problems,' IEEE Signal Process. Mag., vol. 27, no. 3, pp. 20-34, May 2010.

[12] A. Mezghani and J. A. Nossek, "Capacity lower bound of MIMO channels with output quantization and correlated noise," in IEEE Int. Symp. on Inform. Theory Proceedings, 2012.

[13] P. F. Panter and W. Dite, "Quantization distortion in pulse-count modulation with nonuniform spacing of levels," Proceedings of the IRE, vol. 39, no. 1, pp. 44-48, 1951.

[14] T. Wiegand, "Source Coding: Part I of Fundamentals of Source and Video Coding," Foundations and Trends in Signal Process., vol. 4, no. 1-2, pp. 1-222, 2010. [Online]. Available: http://www.nowpublishers.com/article/Details/SIG-010

[15] A. Goldsmith, S. A. Jafar, N. Jindal, and S. Vishwanath, "Capacity limits of MIMO channels," IEEE Journal on Selected Areas in Commun., vol. 21, no. 5, pp. 684-702, Jun. 2003.

[16] O. Orhan, E. Erkip, and S. Rangan, "Low power analog-to-digital conversion in millimeter wave systems: Impact of resolution and bandwidth on performance," in Inform. Theory and Applications Workshop, Feb. 2015, pp. 191-198.

[17] S. N. Diggavi and T. M. Cover, "The worst additive noise under a covariance constraint," IEEE Trans. on Inform. Theory, vol. 47, no. 7 , pp. 3072-3081, Nov. 2001.

[18] S. M. Kogon, "Eigenvectors, diagonal loading and white noise gain constraints for robust adaptive beamforming," in The Thrity-Seventh Asilomar Conf. on Signals, Systems Computers, 2003, vol. 2, Nov. 2003, pp. 1853-1857 Vol.2. 